\title{
ESTUDO DO COMPORTAMENTO REOLÓGICO DE SUCOS COMBINADOS DE FRUTAS VERMELHAS
}

\section{STUDY OF THE RHEOLOGICAL BEHAVIOR OF COMBINED JUICES OF RED FRUITS}

\author{
Charles Windson Isidoro Haminiuk ${ }^{1}$; Maria-Rita Sierakowski ${ }^{2}$; Manuel Salvador Vicente Plata-Oviedo ${ }^{3}$; \\ Ivanise Guilherme Branco ${ }^{4}$; Maria Helene Giovanetti Canteri ${ }^{5}$; Maria Lucia Masson ${ }^{6}$ \\ ${ }^{1}$ Universidade Tecnológica Federal do Paraná - UTFPR - Campo Mourão - Brasil haminiuk@utfpr.edu.br \\ ${ }^{2}$ Universidade Federal do Paraná - UFPR - Curitiba - Brasil mariarita.sierakowski@ ufpr.br \\ ${ }^{3}$ Universidade Tecnológica Federal do Paraná - UTFPR - Campo Mourão - Brasil mapaov@utfpr.edu.br \\ ${ }^{4}$ Universidade Estadual Paulista - UNESP - Assis - Brasil igbranco@ assis.unesp.br \\ ${ }^{2}$ Universidade Tecnológica Federal do Paraná - UTFPR - Ponta Grossa - Brasil canteri@ utfpr.edu.br \\ ${ }^{3}$ Universidade Federal do Paraná - UFPR - Curitiba - Brasil masson@ufpr.br
}

\begin{abstract}
Resumo
O objetivo deste trabalho foi avaliar o comportamento reológico de sucos combinados de morango, amora-preta e framboesa em duas diferentes temperaturas de interesse em processos industriais na indústria de alimentos, usando análises reológicas em regime não-oscilatório e a metodologia de superfície de resposta (MSR). Os dados reológicos foram ajustados pelo modelo de Casson. Todas as amostras de sucos combinados de frutas vermelhas apresentaram um comportamento newtoniano, sendo que os parâmetros reológicos tensão inicial $\left(K_{o c}\right)$ e viscosidade plástica de Casson $\left(K_{o c}\right)$ tiveram uma redução em seus valores com o aumento da temperatura. Pela análise dos diagramas ternários ficou evidente como o suco de framboesa contribuiu efetivamente para os maiores valores da tensão inicial e viscosidade plástica de Casson em ambas as temperaturas estudadas.
\end{abstract}

Palavras-chave: Sucos combinados de frutas vermelhas, reologia, temperatura, MSR.

\section{Introdução}

Morango, amora-preta e framboesa são frutas que pertencem à família das Rosáceas. De acordo com Brown (2002), a família das Rosáceas destaca-se por sua amplitude e diversidade a qual incluiu setenta e cinco gêneros e mais de cento e vinte espécies. Os membros da família das Rosáceas são valorizados pelas suas frutas e produtos derivados. As frutas vermelhas, como são geralmente conhecidas, tem sido freqüentemente usadas pela indústria de alimentos. Seus produtos 
manufaturados, tais como sucos de frutas, néctares, sorvetes e balas, contêm a polpa de fruta como matéria-prima básica (HAMINIUK et al., 2007).

Os sucos de frutas têm uma vida-de-prateleira limitada e são susceptíveis a deterioração microbiana e atividade enzimática, sendo, portanto necessário tratamento térmico para inativar os microorganismos e as enzimas. O desenvolvimento de processos confiáveis e seguros requer conhecimento detalhado do comportamento de fluxo do material a ser escoado. O conhecimento das curvas de fluxos de sucos de frutas é útil no controle de qualidade, controle de processo, calculo da energia utilizada e seleção do equipamento adequado (BELIBAGLI e DALGIC, 2007)

O conhecimento dos parâmetros reológicos é importante em aplicações industriais não somente para determinar o consumo de energia para bombear um suco de fruta com alta viscosidade, mas também para resolver problemas relacionados à incorporação de ar, o que causa dificuldades na operação das bombas e reações indesejadas, tais como oxidação e contaminação. As medidas reológicas têm sido consideradas como uma ferramenta analítica a qual fornece uma compreensão fundamental dentro da organização do alimento (HAMINIUK et al., 2006).

Atualmente, há uma tendência internacional no mercado para novos produtos, tais como sucos combinados, os quais influenciam os fabricantes a desenvolver sucos com dois ou mais tipos de frutas. No entanto, na literatura encontram-se poucos estudos sobre as propriedades físicas e químicas sucos combinados de frutas. Desta forma, o objetivo desta pesquisa foi caracterizar o comportamento reológico de sucos combinados de frutas (misturas ternárias) de morango, framboesa e amora-preta usando a metodologia de superfície de resposta como ferramenta matemática e estatística.

\section{Material e Métodos}

\section{Sucos de frutas vermelhas}

As frutas vermelhas utilizadas neste trabalho foram morango, amora-preta e framboesa. As amostras foram compradas no mercado local da cidade de Curitiba, estado do Paraná. Após o despolpamento, os sucos das frutas vermelhas foram obtidos utilizando uma centrífuga (Sorval), na qual as polpas integrais foram centrifugadas a 9000 RPM por 20 minutos. Utilizou-se o sobrenadante (suco) para a realização das análises e obtenção das misturas.

\section{Análises físico-químicas dos sucos de frutas vermelhas}

Os sólidos solúveis totais ( ${ }^{\circ} \mathrm{Brix}$ ) e $\mathrm{pH}$ foram determinados usando um refratômetro (WY1A; ABBE, USA) e pHmetro (model $710 \mathrm{~A}$; Orion Research, Boston, USA) à $25^{\circ} \mathrm{C}$, respectivamente. A 
acidez titulável e a umidade dos sucos foram medidas de acordo com a metodologia descrita na A.O.A.C. (2000). Os resultados das análises físico-químicas podem ser visualizados na Tabela 2.

\section{Formulação e delineamento experimental}

As proporções dos sucos combinados (misturas ternárias) foram definidas através a Metodologia de Superfície de Resposta (MSR) utilizando um delineamento simplex-centróide expandido de 10 tratamentos, com o objetivo de obter as formulações. Todas as 10 combinações foram obtidas em triplicata. A Tabela 1 apresenta o delineamento estatístico usado para a formulação dos sucos combinados de frutas vermelhas.

Tabela 1 - Delineamento simplex-centróide expandido de 10 tratamentos para os sucos combinados de

\begin{tabular}{cccc}
\multicolumn{4}{c}{ morango, amora-preta e framboesa } \\
\hline Formulações & $\mathrm{X}^{\prime} 1$ & $\mathrm{X}^{\prime} 2$ & $\mathrm{X}^{\prime} 3$ \\
1 & 1 & 0 & 0 \\
2 & 0 & 1 & 0 \\
3 & 0 & 0 & 1 \\
4 & $1 / 2$ & $1 / 2$ & 0 \\
5 & $1 / 2$ & 0 & $1 / 2$ \\
6 & 0 & $1 / 2$ & $1 / 2$ \\
7 & $1 / 3$ & $1 / 3$ & $1 / 3$ \\
8 & $2 / 3$ & $1 / 6$ & $1 / 6$ \\
9 & $1 / 6$ & $2 / 3$ & $1 / 6$ \\
10 & $1 / 6$ & $1 / 6$ & $2 / 3$ \\
\hline
\end{tabular}

X'1 - Suco de Morango. X’2 - Suco de Amora-Preta. X’3 - Suco de Framboesa

As respostas avaliadas para cada formulação foram a tensão inicial $\left(\mathrm{K}_{\mathrm{oc}}\right)$ e a viscosidade plástica $\left(\mathrm{K}_{\mathrm{c}}\right)$, onde foi utilizado o modelo de Casson para ajustar as curvas de fluxo. A análise estatística foi conduzida utilizando o valor de cada replicata com o objetivo de se obter a análise de falta de ajuste (lack-of-fit). A Metodologia de Superfície de Resposta permite a modelagem dos resultados usando equações de segunda-ordem. Modelos de regressão múltipla [quadrático (equação 1) e cúbico especial (equação 2)] foram utilizados para os dois parâmetros reológicos estudados pelo modelo de Casson.

$$
\begin{array}{ll}
Y=\beta_{1}^{\prime} X_{1}^{\prime}+\beta_{2}^{\prime} X_{2}^{\prime}+\beta_{3}^{\prime} X_{3}^{\prime}+\beta_{1}^{\prime} \beta_{2}^{\prime} X_{1}^{\prime} X_{2}^{\prime}+\beta_{1}^{\prime} \beta_{3}^{\prime} X_{1}^{\prime} X_{3}^{\prime}+\beta_{2}^{\prime} \beta_{3}^{\prime} X_{2}^{\prime} X_{3}^{\prime} & \text { Eq. } 1 \\
Y=\beta_{1}^{\prime} X_{1}^{\prime}+\beta_{2}^{\prime} X_{2}^{\prime}+\beta_{3}^{\prime} X_{3}^{\prime}+\beta_{1}^{\prime} \beta_{2}^{\prime} X_{1}^{\prime} X_{2}^{\prime}+\beta_{1}^{\prime} \beta_{3}^{\prime} X_{1}^{\prime} X_{3}^{\prime}+\beta_{2}^{\prime} \beta_{3}^{\prime} X_{2}^{\prime} X_{3}^{\prime}+\beta_{1}^{\prime} \beta_{2}^{\prime} \beta_{3}^{\prime} X_{1}^{\prime} X_{2}^{\prime} X_{3}^{\prime} & \text { Eq. } 2
\end{array}
$$

onde, Y é a variável reológica, $\beta$ é o coeficiente gerado pela regressão múltipla e $\mathrm{X}$ é a proporção dos sucos de frutas vermelhas.

\section{Medidas reológicas}

O estudo do comportamento de fluxo dos sucos combinados de frutas vermelhas (misturas ternárias) obtidos por delineamento experimental em regime não-oscilatório foi realizado em um reômetro Haake Rheostress 600 (Haake, Karlsruhe, Alemanha) (CHAKRANBANDHU e SING, 
2005), onde foi utilizado um sensor tipo cone-placa $\left(\mathrm{C}-60 / 2^{\circ} \mathrm{Ti}\right.$, com abertura de $\left.100 \mu \mathrm{m}\right)$. Um banho termostático Haake DC-30 e um sistema de controle de temperatura universal Haake (Haake, Karlsruhe, Alemanha) foram utilizados para ajustar a temperatura das misturas ternárias nas temperaturas de $20{ }^{\circ} \mathrm{C}$ e $60{ }^{\circ} \mathrm{C}$. Para a realização das análises reológicas, foi utilizado para cada medida $1 \mathrm{~mL}$ de amostra (de acordo com orientação do fabricante) sendo esta mantida por um minuto antes da análise para estabilização da temperatura. A amostra não foi reutilizada após o aquecimento, devido às mudanças nas propriedades reológicas (HAMINIUK et al., 2009). Para cada corrida experimental a curva ascendente teve uma duração de dois minutos (correspondente a 4,8 segundos x 25 pontos), com a taxa de cisalhamento variando de 0 a $300 \mathrm{~s}^{-1}$ e dois minutos para a curva descendente com a taxa de cisalhamento variando de 300 a $0 \mathrm{~s}^{-1}$. Para ambas as curvas (ascendente e descendente), obtiveram-se 25 pontos de tensão de cisalhamento versus taxa de cisalhamento, resultando em 50 pontos, dos quais foi calculado o valor médio da tensão de cisalhamento para cada taxa de cisalhamento. Três corridas experimentais foram feitas para cada mistura ternária obtida pelo delineamento experimental, e a tensão de cisalhamento resultante foi à média de três valores experimentais (FONSECA et al., 2009). O efeito de tixotropia não foi observado nas misturas ternárias. Os dados reológicos de taxa de cisalhamento e tensão de cisalhamento dos sucos combinados de frutas vermelhas foram ajustados ao modelo de Casson.

\section{Análise Estatística}

Os dados reológicos foram ajustados ao modelo de Casson utilizando o software Origin (OriginLab Corporation, Ma, USA) e a análise estatística das respostas reológicas obtidas pelo modelo de Casson $\left(\mathrm{K}_{\mathrm{oc}}\right.$ e $\mathrm{K}_{\mathrm{c}}$ ) foi conduzida utilizando o software Statistica (Statsoft, Tulsa, OK, USA). Os dados obtidos foram submetidos à análise de variância e a diferença entre as médias foi analisada através do teste de Tukey ao nível de significância de 5\%.

\section{Resultados e Discussão}

Com relação às características físico-químicas das polpas integrais e dos sucos integrais pode-se observar que não houve uma variação considerável com o processo de remoção dos sólidos insolúveis (Tabela 2). Recentemente Vidal et al.(2006) estudaram as características reológicas da polpa de manga na forma integral e centrifugada e de acordo com este estudo não houve diferença significativa nos valores de $\mathrm{pH}$ e sólidos solúveis com o processo de centrifugação. No entanto, em relação aos sólidos insolúveis houve diferença significativa $(\mathrm{P} \leq 0,05)$. 
Tabela 2 - Caracterização físico-química das polpas integrais e centrifugadas das polpas de frutas vermelhas

\begin{tabular}{cccc}
\hline Parâmetros & Polpa de Morango & Polpa de Amora-Preta & Polpa de Framboesa \\
\hline \multicolumn{3}{c}{ Integrais } \\
\hline Sólidos Solúveis ( ${ }^{\circ}$ Brix) & 6,10 & 5,75 & 6,95 \\
$\mathrm{pH}$ & 3,60 & 3,60 & 3,30 \\
Acidez Titulável (g/100ml*) & 0,69 & 0,57 & 1,09 \\
Umidade $(\%)^{\#}$ & 92,48 & 93,15 & 92,10 \\
\hline Sólidos Solúveis ( ${ }^{\circ}$ Brix) & \multicolumn{2}{c}{ Centrifugadas } & 6,90 \\
$\mathrm{pH}$ & 6,08 & 5,75 & 3,35 \\
Acidez Titulável (g/100ml) & 3,58 & 3,62 & 1,00 \\
\hline
\end{tabular}

* Expresso em ácido cítrico. ${ }^{\#}$ Análise não realizada para a polpa centrifugada

Reologia não-oscilatória-curvas de fluxo

As curvas de fluxo dos sucos combinados nas temperaturas de $20{ }^{\circ} \mathrm{C}$ e $60{ }^{\circ} \mathrm{C}$ estão apresentadas nas Figuras 1 e 2, sendo que para a obtenção dessas curvas e os diagramas ternários obtidos pela Metodologia de Superfície de Resposta (MSR), extraiu-se a raiz quadrada dos dados de taxa de cisalhamento e tensão de cisalhamento, de acordo com a equação proposta por Casson (1959). Os pontos marcados representam os valores médios dos dados experimentais (reogramas) e as linhas contínuas é o resultado do ajuste pelo modelo de Casson. O modelo de Casson foi anteriormente utilizado por Branco (2001) no ajuste de dados reológicos, onde apresentou um bom ajuste aos dados experimentais de misturas ternárias combinando polpa de manga com suco de cenoura e de laranja.

Observando a relação entre a taxa de cisalhamento e tensão de cisalhamento nas Figuras 1 e 2, fica claro um comportamento newtoniano dos sucos combinados de frutas vermelhas.

Figura 1 - Curvas de fluxo dos sucos combinados de morango, framboesa e amora-preta ajustadas pelo modelo de Casson na temperatura de $20^{\circ} \mathrm{C}$. Sensor de medida C-60/2 $\mathrm{Ti}$, com abertura de $100 \mu \mathrm{m}$

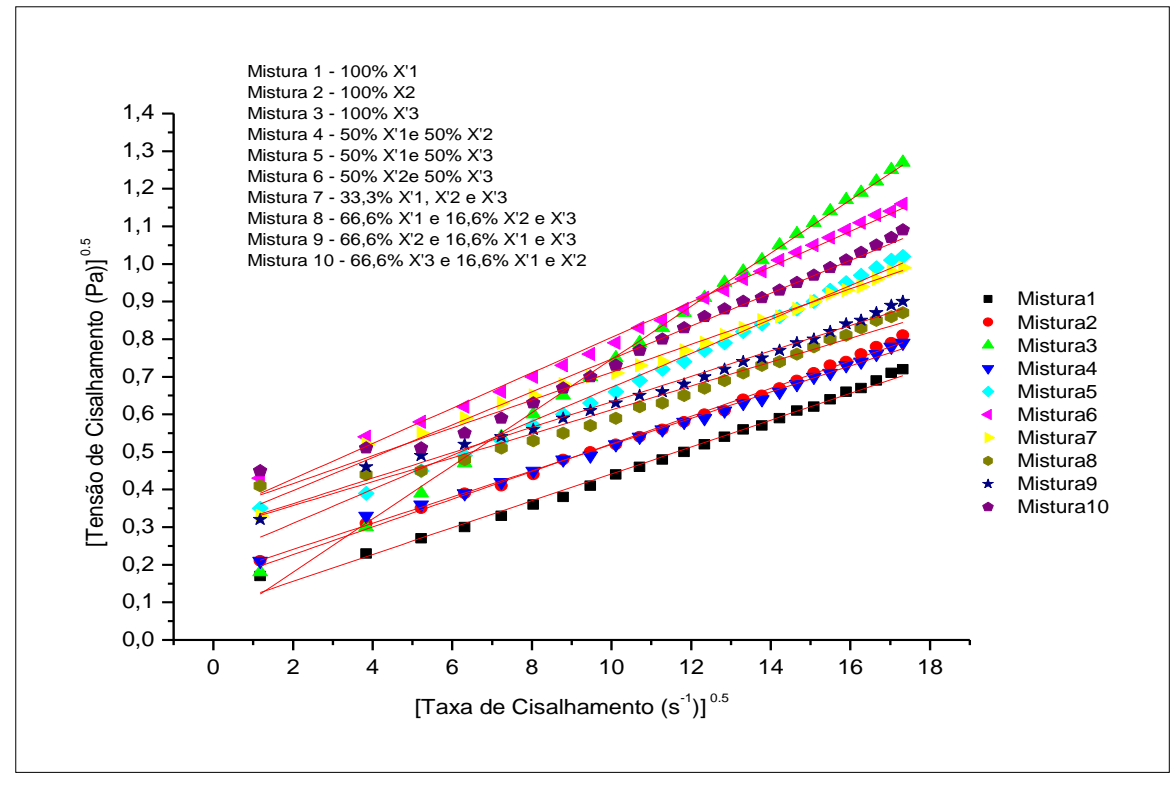


Segundo Scharamm (2000) as propriedades reológicas de fluidos newtonianos são independentes da taxa de cisalhamento e do histórico anterior do cisalhamento e dependente somente da composição e da temperatura. A independência dos valores de tensão de cisalhamento $(\tau)$ em relação à taxa de cisalhamento $(\gamma)$ demonstra a característica newtoniana dos sucos combinados de frutas vermelhas. Os reogramas ajustados pelo modelo de Casson apresentaram altos valores de coeficiente de determinação $\left(\mathrm{R}^{2}\right)$ e baixos valores de chi-quadrado $\left(\chi^{2}\right)$, sendo que na temperatura de $20{ }^{\circ} \mathrm{C}$ os valores de $\mathrm{R}^{2}$ variaram entre 0,96 a 0,99 .

Figura 2 - Curvas de fluxo dos sucos combinados de morango, framboesa e amora-preta ajustadas pelo modelo de Casson na temperatura de $60{ }^{\circ} \mathrm{C}$. Sensor de medida C-60/2 ${ }^{\circ} \mathrm{Ti}$, com abertura de $100 \mu \mathrm{m}$

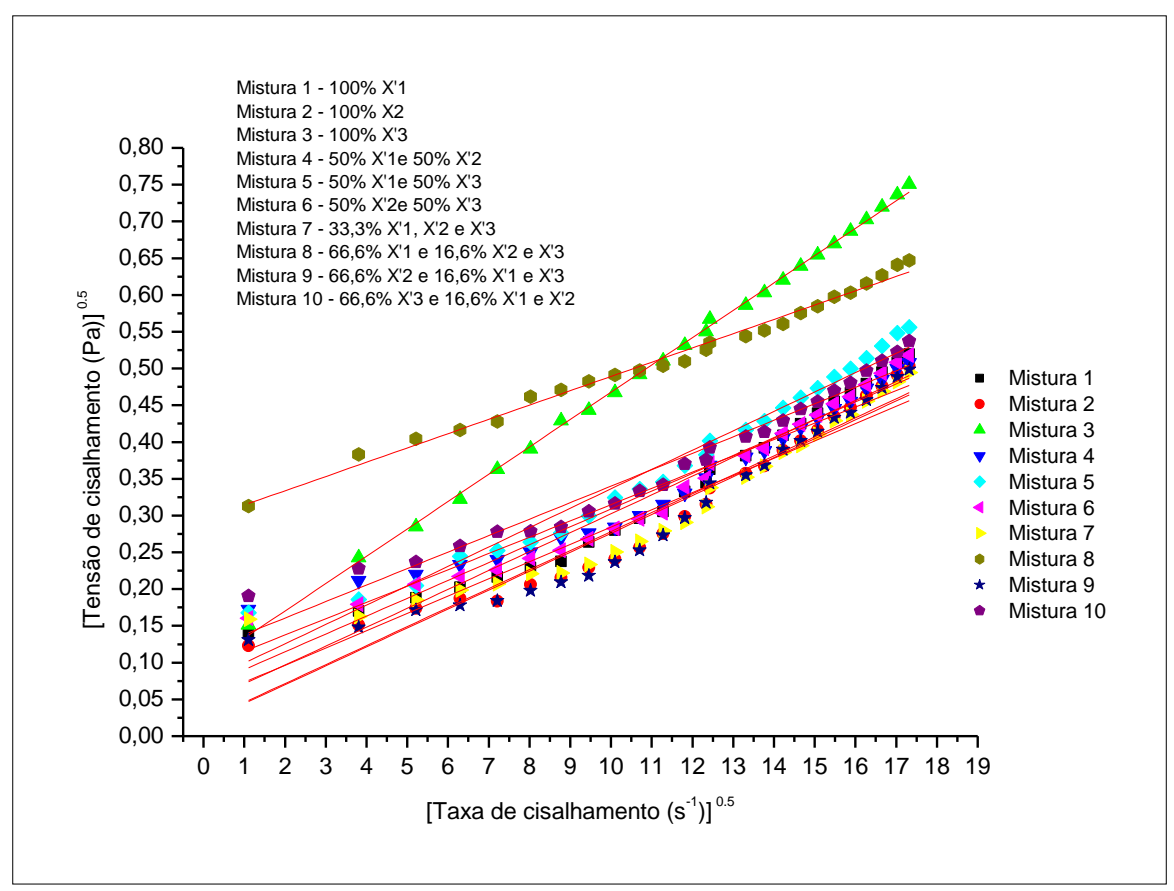

Os valores dos coeficientes de determinação $\left(\mathrm{R}^{2}\right)$ à $60{ }^{\circ} \mathrm{C}$ para os sucos combinados (misturas ternárias) variaram entre 0,91 a 0,99 . No entanto, pode-se observar algumas anormalidades nos valores de tensão de cisalhamento com o aumento progressivo da temperatura. Típicos exemplos associados ao aquecimento incluem o amolecimento de chocolates ou de queijos devido ao derretimento da gordura, geleificação para formar géis pécticos, espessamento de soluções devido à gelatinização do amido e enrijecimento da carne e produtos derivados de ovos causados pela desnaturação das proteínas (STEFFE, 1996).

Neste estudo essas anormalidades são devido a duas possíveis causas: o aumento da temperatura, o que causa uma expansão térmica na amostra tornando o reograma menos constante devido à dificuldade do alinhamento das moléculas durante a curva de descida (durante a obtenção da curva de fluxo); outra causa possível, é que devido a baixa viscosidade das misturas centrifugadas, em torno de 0,03 Pa.s, aliado a expansão térmica gerada pela temperatura, o torque mínimo do reômetro não é superado pela resistência natural das mistura ternárias, fazendo que 
durante a obtenção da curva de volta (curva de descida) ocorra valores de tensão de cisalhamento nulos. Durante a obtenção dos reogramas, portanto, observa-se uma perturbação na linearidade das curvas de fluxo para as misturas ternárias em alta temperatura.

As Tabelas 3 e 4 apresentam os valores das resposta ajustadas ao modelo de Casson. Comportamento fora do padrão para a tensão inicial pode ser observado nas formulações 1 (100\% suco de morango) e 3 (100\% suco de framboesa) de acordo com a Figura 3.

Com o aumento da temperatura ocorreu um aumento na tensão inicial, embora não tenha havido diferença significativa $(\mathrm{P}>0,05)$ nas duas temperaturas estudadas nessas duas formulações, diferentemente das outras formulações, onde com o aumento da temperatura, ocorreu uma redução significativa no parâmetro $\mathrm{K}_{\mathrm{oc}}$. $\mathrm{Na}$ temperatura de $20{ }^{\circ} \mathrm{C}$, a formulação 7 apresentou o maior valor de tensão inicial (33\% de cada suco), enquanto que a formulação 1 apresentou o menor valor de $\mathrm{K}_{\mathrm{oc}}$.

Tabela 3 - Delineamento simplex-centroide expandido de 10 tratamentos para os sucos combinados de morango, framboesa e amora-preta a $20^{\circ} \mathrm{C}$

\begin{tabular}{lccccc}
\hline Misturas & \multicolumn{2}{c}{ Componentes Originais (codificados) } & \multicolumn{2}{c}{ Respostas } \\
\hline & $\mathrm{X}^{\prime} 1$ & $\mathrm{X}^{\prime} 2$ & $\mathrm{X}^{\prime} 3$ & $\mathrm{~K}_{\mathrm{oc}}$ & $\mathrm{K}_{\mathrm{c}}$ \\
1 & 1 & 0 & 0 & 0,03 & 35,56 \\
2 & 0 & 1 & 0 & 0,15 & 37,96 \\
3 & 0 & 0 & 1 & 0,05 & 70,55 \\
4 & $1 / 2$ & $1 / 2$ & 0 & 0,19 & 32,99 \\
5 & $1 / 2$ & 0 & $1 / 2$ & 0,20 & 44,91 \\
6 & 0 & $1 / 2$ & 0,29 & 43,86 \\
7 & $1 / 3$ & $1 / 3$ & 0,38 & 33,51 \\
8 & $2 / 3$ & $1 / 6$ & $1 / 3$ & 0,29 & 30,05 \\
9 & $1 / 6$ & $2 / 3$ & $1 / 6$ & 0,29 & 33,71 \\
10 & $1 / 6$ & $1 / 6$ & $2 / 3$ & 0,31 & 43,79 \\
\hline
\end{tabular}

X’1 - Polpa de morango. X’2 - Polpa de amora-preta. X’3 - Polpa de framboesa. $K_{o c}$ (Tensão inicial - Pa) e $K_{c}$ (Viscosidade plástica de Casson mPa.s). Os dados reológicos foram obtidos pelo modelo de Casson.

Tabela 4 - Delineamento simplex-centroide expandido de 10 tratamentos para os sucos combinados de morango, framboesa e amora-preta a $60^{\circ} \mathrm{C}$

\begin{tabular}{cccccc}
\hline Misturas & \multicolumn{2}{c}{ Componentes Originais (codificados) } & \multicolumn{2}{c}{ Respostas } \\
\hline & $\mathrm{X}^{\prime} 1$ & $\mathrm{X}^{\prime} 2$ & $\mathrm{X}^{\prime} 3$ & $\mathrm{~K}_{\mathrm{oc}}$ & $\mathrm{K}_{\mathrm{c}}$ \\
1 & 1 & 0 & 0 & 0,05 & 21,06 \\
2 & 0 & 1 & 0 & 0,02 & 25,82 \\
3 & 0 & 0 & 1 & 0,11 & 37,17 \\
4 & $1 / 2$ & $1 / 2$ & 0 & 0,04 & 22,15 \\
5 & $1 / 2$ & 0 & $1 / 2$ & 0,05 & 26,34 \\
6 & 0 & $1 / 2$ & $1 / 2$ & 0,02 & 24,28 \\
7 & $1 / 3$ & $1 / 3$ & $1 / 3$ & 0,04 & 23,46 \\
8 & $2 / 3$ & $1 / 6$ & $1 / 6$ & 0,03 & 17,06 \\
9 & $1 / 6$ & $2 / 3$ & $1 / 6$ & 0,01 & 25,73 \\
10 & $1 / 6$ & $1 / 6$ & $2 / 3$ & 28,28 \\
\hline
\end{tabular}

X’1 - Polpa de morango. X’2 - Polpa de amora-preta. X’3 - Polpa de framboesa. $K_{o c}$ (Tensão inicial - Pa) e $K_{c}$ (Viscosidade plástica de Casson mPa.s). Os dados reológicos foram obtidos pelo modelo de Casson.

No entanto, para a temperatura de $60{ }^{\circ} \mathrm{C}$ não houve variações muito significativas nos valores de $\mathrm{K}_{\mathrm{oc}}$, ficando na faixa de 0,02 a 0,11 Pa. Em relação à viscosidade plástica de Casson 
(Figura 4) ao nível de significância de 95\%, houve diferença significativa nos valores de $\mathrm{K}_{\mathrm{c}}$ com o aumento da temperatura de acordo com o teste de Tukey.

Segundo a Figura 4, pode-se observar perfil similar dos sucos combinados com o aumento da temperatura, sendo que as formulações 3 (100\% de suco de framboesa) e 8 (66\% de suco de morango e $17 \%$ de sucos de amora-preta e framboesa) apresentaram o maior e menor valor de $\mathrm{K}_{\mathrm{C}}$ na temperatura de $20{ }^{\circ} \mathrm{C}$ e $60{ }^{\circ} \mathrm{C}$, respectivamente.

Figura 3- Efeito da temperatura na tensão inicial nos sucos combinados de morango, framboesa e amora-preta

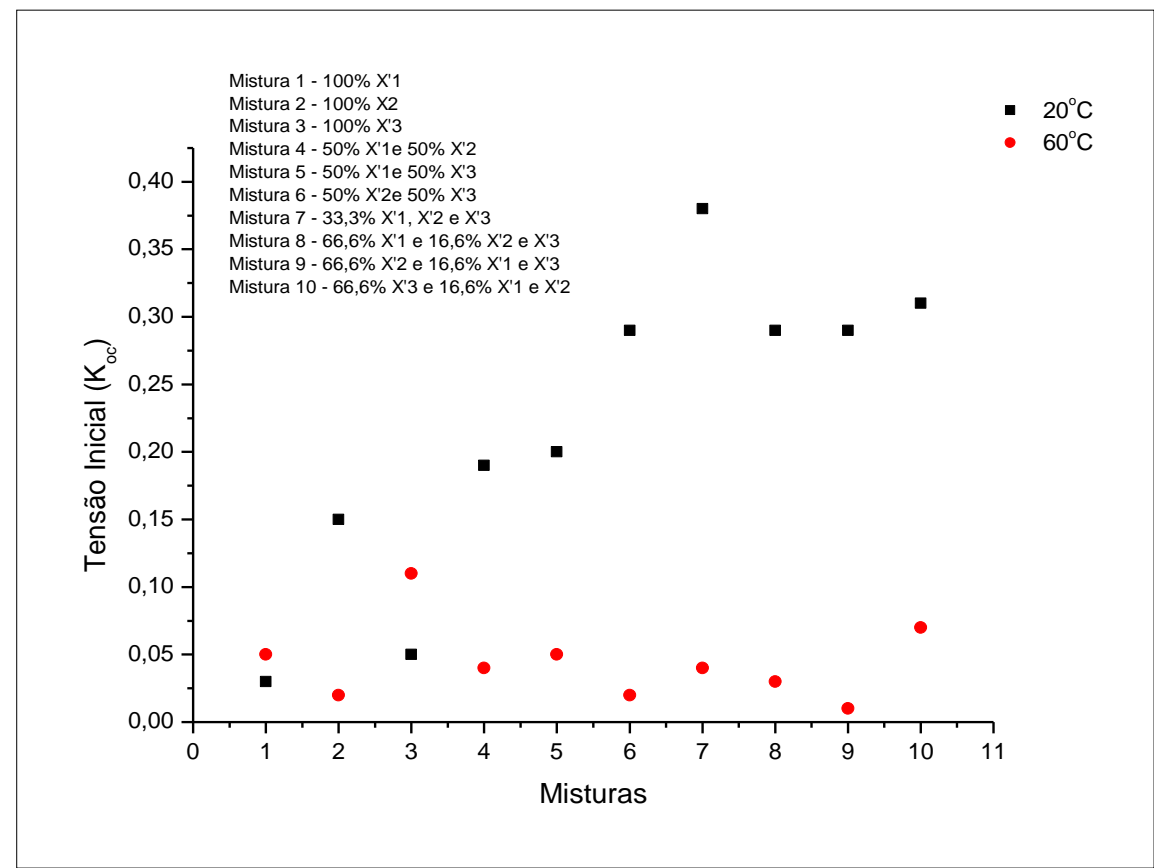

Figura 4. Efeito da temperatura na viscosidade plástica de Casson nos sucos combinados de morango, framboesa e amora-preta.

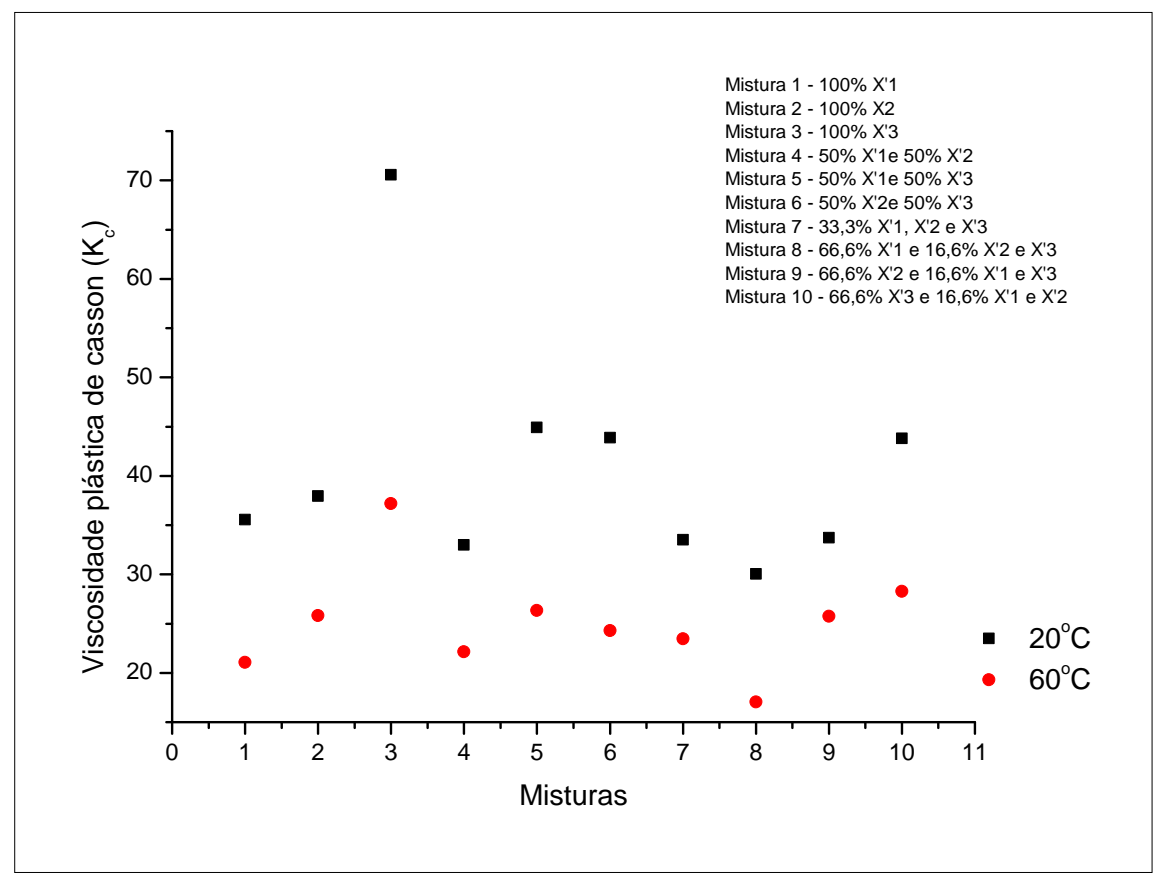


Diagramas ternários dos sucos combinados a $20^{\circ} \mathrm{C}$

A representação do ajuste cúbico ao sistema ternário para a tensão inicial $\left(\mathrm{K}_{\mathrm{oc}}\right)$ está representada na Figura 5. Neste caso, as formulações com 100\% de sucos de frutas vermelhas não apresentaram efeito relevante nos valores da tensão inicial para as misturas ternárias. Segundo a Figura 5, os maiores valores de $\mathrm{K}_{\mathrm{oc}}$ podem ser visualizados no interior do triângulo, demonstrando que na temperatura de $20{ }^{\circ} \mathrm{C}$ a combinação entre os três sucos apresenta maior influência em $\mathrm{K}_{\mathrm{oc}}$ do que as frações individuais dos sucos. O modelo cúbico especial foi escolhido para o ajuste dos dados reológicos para a tensão inicial $\left(\mathrm{K}_{\mathrm{oc}}\right)$ devido este ter apresentado alto valor de coeficiente de determinação $\left(R^{2}=0,93\right)$ e baixo valor de erro puro de 0,018. A análise de falta de ajuste não foi significativa $(\mathrm{P}>0,05)$ demonstrando que o modelo obtido é preditivo e estatisticamente aceitável.

Figura 5 - Diagrama ternário para a tensão inicial $\left(\mathrm{K}_{\mathrm{oc}}\right)$ na temperatura de $20^{\circ} \mathrm{C}$ ajustado pelo modelo cúbico especial

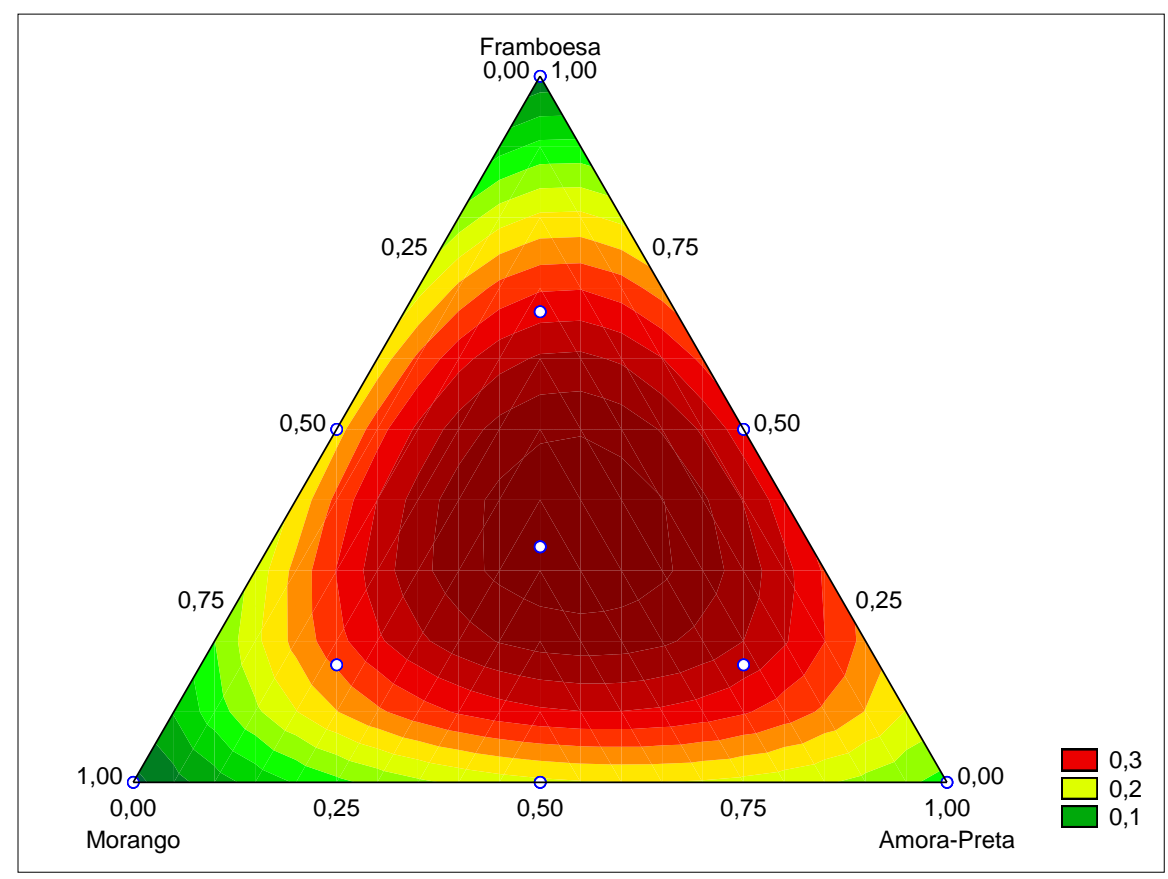

Através da análise da Figura 6 (diagrama ternário à $20{ }^{\circ} \mathrm{C}$ ) fica evidente o efeito que a fração X’3 teve na viscosidade plástica de Casson, demonstrando que quanto maior for a fração do suco de framboesa maior será o valor de $\mathrm{K}_{\mathrm{c}}$. Não houve diferença estatística ao nível de $95 \%$ de probabilidade relacionado à influência das frações X'1 e X'2 na mistura ternária, demonstrando que essas frações apresentam o mesmo efeito. Através da análise de variância (dados não apresentados) pode-se observar que o modelo cúbico especial representou bem os dados de $\mathrm{K}_{\mathrm{c}}$ devido ao alto valor do coeficiente de determinação $\left(\mathrm{R}^{2}=0,95\right)$. A análise de falta de ajuste não foi significativa $(\mathrm{P}>0,05)$, demonstrando que os dados experimentais apresentaram uma boa repetibilidade $(\mathrm{P}=0,08)$. 
Figura 6- Diagrama ternário para a viscosidade plástica de casson $\left(\mathrm{K}_{\mathrm{c}}\right)$ na temperatura de $20{ }^{\circ} \mathrm{C}$ ajustado pelo modelo cúbico especial.

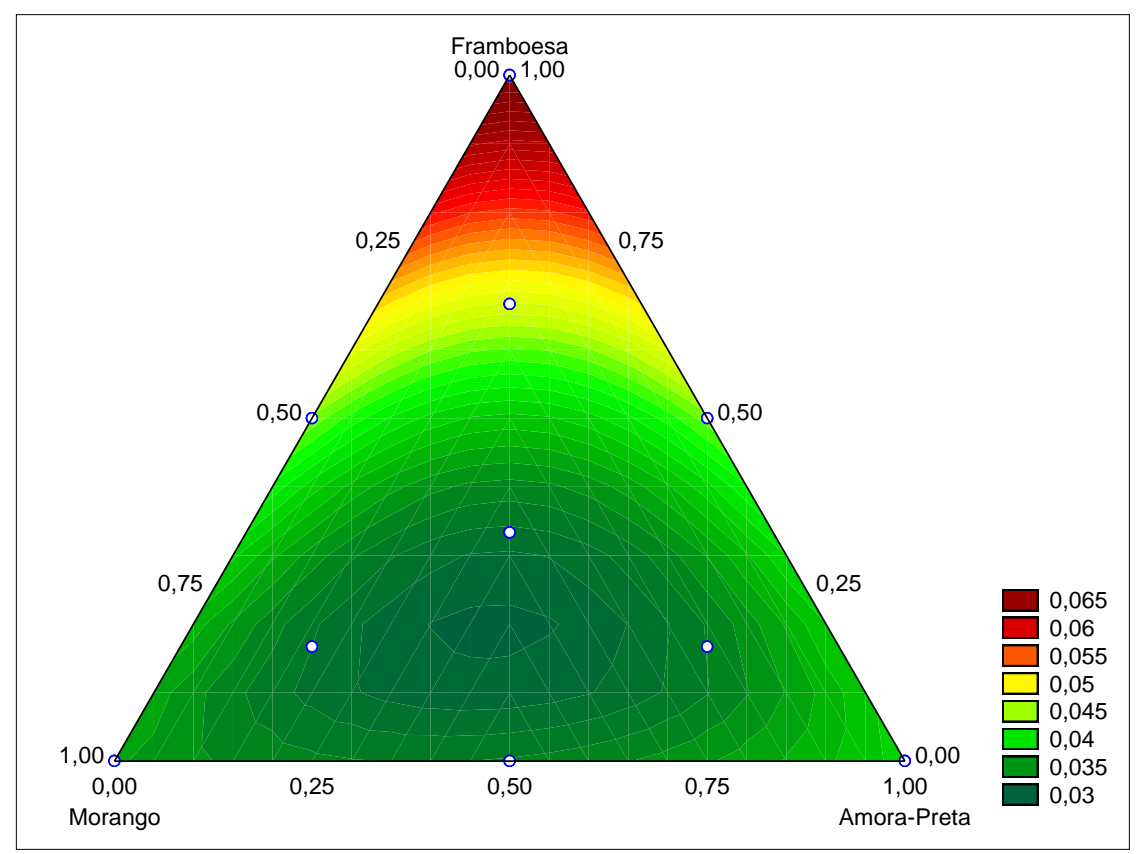

Diagramas ternários dos sucos combinados a $60^{\circ} \mathrm{C}$

Com o aumento da temperatura houve variação no comportamento dos sucos combinados quando comparado com a temperatura de $20{ }^{\circ} \mathrm{C}$. Na Figura 7 encontra-se representado o ajuste com o modelo quadrático aos dados experimentais para a tensão inicial à $60{ }^{\circ} \mathrm{C}$. De acordo com o diagrama ternário dos sucos combinados de frutas vermelhas, o suco de amora-preta contribui para os menores valores de $\mathrm{K}_{\mathrm{oc}}$, enquanto que o suco de framboesa colabora para os maiores valores de $\mathrm{K}_{\mathrm{oc}}$, ficando o suco de morango em um valor intermediário. $\mathrm{O}$ modelo quadrático foi o que apresentou um melhor ajuste aos dados experimentais, com valor de $\mathrm{R}^{2}$ de 0,81 e valor de erro puro próximo de zero, sendo que a falta de ajuste devido às replicatas não foi significativa $(\mathrm{P}>0,05)$.

Figura 7- Diagrama ternário para a tensão inicial $\left(\mathrm{K}_{\mathrm{oc}}\right)$ na temperatura de $60{ }^{\circ} \mathrm{C}$ ajustado pelo modelo quadrático.

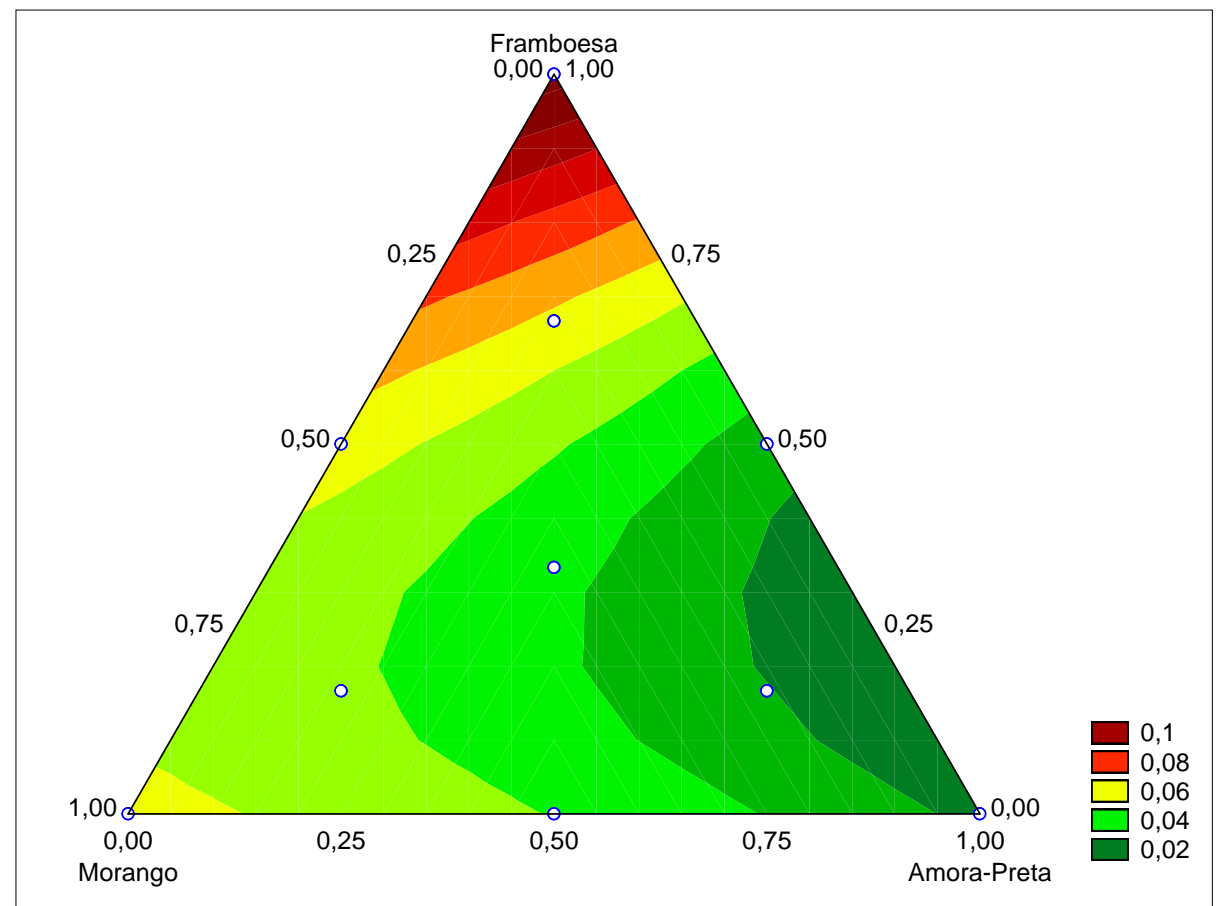


Figura 8- Diagrama ternário para a viscosidade plástica de casson $\left(\mathrm{K}_{\mathrm{c}}\right)$ na temperatura de $60{ }^{\circ} \mathrm{C}$ ajustado pelo modelo quadrático

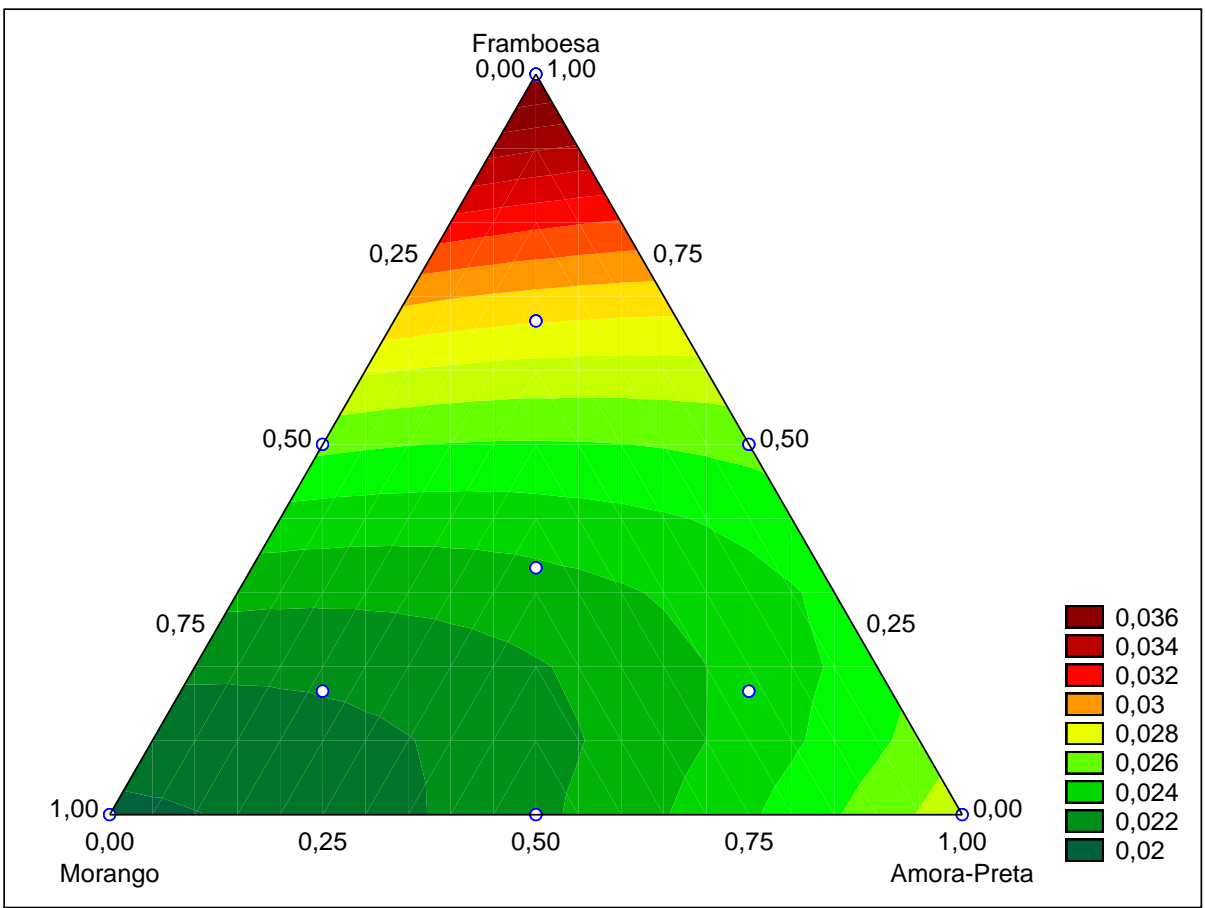

A Figura 8 apresenta o diagrama ternário para a viscosidade plástica de Casson obtida na temperatura de $60{ }^{\circ} \mathrm{C}$ para os sucos de frutas vermelhas. O ajuste dos dados reológicos ao modelo quadrático resultou em um coeficiente de determinação de 0,75. Comparando as Figuras $8\left(60{ }^{\circ} \mathrm{C}\right) \mathrm{e}$ $6\left(20{ }^{\circ} \mathrm{C}\right)$ pode-se observar que não houve uma mudança significativa em relação ao efeito da temperatura nos sucos combinados, exercendo a fração de framboesa em ambas as temperaturas estudadas um maior efeito na viscosidade plástica de Casson, quando comparada com as outras frações.

\section{Conclusão}

Em relação aos dados reológicos dos sucos combinados de frutas vermelhas ajustados pelo modelo reológico de Casson, um comportamento newtoniano foi observado. Este comportamento justifica-se pela remoção dos sólidos insolúveis durante o processo de centrifugação. Ocorreu uma redução dos parâmetros reológicos tensão inicial $\left(\mathrm{K}_{\mathrm{oc}}\right)$ e viscosidade plástica de Casson $\left(\mathrm{K}_{\mathrm{c}}\right)$ com o aumento da temperatura conforme esperado. De acordo com os diagramas ternários obtidos pela Metodologia de Superfície de Resposta (MSR), os modelos quadráticos e cúbicos especial ajustaram bem os dados experimentais, sendo que o suco de framboesa contribuiu efetivamente para os maiores valores da tensão inicial e viscosidade plástica de Casson em ambas as temperaturas estudadas. 


\begin{abstract}
The aim of this work was to evaluate the rheological behavior of combined juices of strawberry, blackberry and raspberry in two different temperatures of interest in industrial processes in the food industry, using rheological analyses on non-oscillatory regime and the Response Surface Methodology (RSM). The rheological data were fitted by the Casson model. All samples of combined juices of ref fruits showed a Newtonian behavior and the rheological parameters yield stress $\left(K_{c}\right)$ and plastic viscosity of Casson $\left(K_{o c}\right)$ had a decrease in its values with the temperature increase. By the analysis of the ternary diagrams it is evident that the raspberry juice effectively contributed to the higher values of yield stress and plastic viscosity of Casson in both temperatures studied.
\end{abstract}

Key-words: Combined juices of red fruits, rheology, temperature, RSM.

\title{
Referências
}

AOAC. Official Methods of Analysis. 17th ed. Gaithersburg, Maryland, USA, AOAC International, 2000.

BELIBAGLI, K. B.; DALGIC, A. C. Rheological properties of sour-cherry juice and concentrate. International Journal of Food Science and Technology, v. 42, n. 6, p. 773-776, 2007. D.O.I:10.1111/j.1365-2621.2007.01578.x

BRANCO, I. G.Tese de Doutorado. Universidade Estadual de Campinas, Campinas, Brasil, 2001.

BROWN, D. The Royal Horticultural Society. New Encyclopedia of Herbs and their Uses. London: Dorling Kindersley, 2002.

CASSON, N. A. Flow equation for pigmento-oil suspensions of the printing ink type, in Rheology of Disperse Suspensions. C.C. Mill (Ed.) Pergamon press, New York, 1959.

CHAKRABANDHU, K.; SINGH, R. K. Rheological properties of coarse food suspensions in tube flow at high temperatures. Journal of Food Engineering, v. 66, n. 1, p. 117-128, 2005. D.O.I: 10.1016/j.jfoodeng.2004.02.039

FONSENSA, V. C.; HAMINIUK, C. W. I.; IZIDORO, D. R.; WASZCZYNSKYJ, N.; SCHEER, A. P.; SIERAKOWSKI, M-R. Stability and rheological behaviour of salad dressing obtained with whey and different combinations of stabilizers. International Journal of Food Science and Technology, v. 44, n. 4, p. 777-783, 2009. D.O.I:10.1111/j.1365-2621.2008.01897.x

HAMINIUK C. W. I.; SIERAKOWSKI M.-R.; BRANCO I. G.; MACIEL G. M.; MASSON, M. L.Rheological study of ternary mixtures and pectic gels of red fruit pulps, International Journal of Food Science and Technology, v. 42, n. 6, p. 629-639, 2007. D.O.I: 10.1111/j.1365-2621.2006.01311.x

HAMINIUK, C. W. I.; SIERAKOWSKI, M.-R.; VIDAL, J. R. M. B.; MASSON, M. L. Influence of temperature on rheological behaviour ofwhole Araçá pulp (Psidium cattleianum sabine). Lebensmittel-Wissenschaft und Lebensmittel-Wissenschaft und Technologie - Food Science and Technology, v. 39, n. 4, p. 426-430, 2006.

HAMINIUK, C. W. I.; SIERAKOWSKI, M-R.; IZIDORO, D. R.; MACIEL, G. M.; SCHEER, A. P.; MASSON, M. L. Comportamento reológico de sistemas pécticos de polpas de frutas vermelhas. Ciência Tecnologia de Alimentos, v. 29, n. 1, p. 225-231, 2009. D.O.I: 10.1590/S0101-20612009000100035

SCHARAMM, G. A pratical approach to rheology and rheometry. 2 ed. Karlsruhe, Germany: [s.n.], 2000.

STEFFE, J. F. Rheological methods in food process engineering (2nd ed). MI: Freeman Press, 1996.

VIDAL, J. R. M. B.; SIERAKOWSKI, M-R; HAMINIUK, C. W. I.; MASSON, M. L.Propriedades reológicas da polpa de manga (Mangifera indica L. cv. Keitt) centrifugada. Ciência e Agrotecnologia [online]. v. 30, n. 5, p. 955-960, 2006. D.O.I:10.1590/S1413-70542006000500020

Submetido em 18 out. 2010; Revisão em 18 jan. 2011; Aceito para publicação em 30 jun.2011. 